\title{
Efecto antioxidante del yodo como protector de la cardiotoxicidad inducida por doxorrubicina y 5-fiuoracilo
}

Reyes Amador M. A*. Gómez Hernández $G^{*}$, Aceves Velasco C.Y**, Alfaro-Hernández Y**, Delgado $G^{* *}$.

\section{Resumen}

- En el presente trabajo valoramos el efecto antioxi-

- dante del yodo y su posible uso como protector de

- la cardiotoxicidad causada por doxorrubicina y 5-flo-

- uracilo. La doxorrubicina causa lesiones cardíacas por

- el exceso de producción de radicales libres. El meca-

- nismo de daño cardiaco por 5-fluoracilo no es claro,

- sin embargo creemos que los radicales libres podrían

- estar involucrados también en la toxicidad producida

- por este fármaco. Utilizamos ratas hembra Sprague-

- Dawley a las que se les administró yodo molecular

- por ocho días consecutivos, a través de una solución

- de lugol al 0.05\% ad limitum; a los tres y siete días

- se les administró una dosis de un mg intraperitoneal

- de doxorrubicina para un grupo y cuatro dosis con-

- tinuas de un mg intraperitoneal de 5-fluoracilo para

otro grupo. Se cuantificó la actividad de catalasa, la lipoperoxidación y la expresión de p53. Los grupos que recibieron la quimioterapia aumentaron los niveles de lipoperoxidación y disminuyeron los de catalasa; además en el grupo de doxorrubicina se observó una tendencia a contrarrestar estos efectos lo que no se observó con el grupo de 5-fluoracilo mas yodo. En la expresión de p53 los grupos de la quimioterapia aumentan su expresión y los que adicionalmente recibieron el yodo tienden a disminuirla. Finalmente podemos concluir que el yodo a través de mecanismos poco claros funciona como un potente antioxidante y que puede reducir la cardiotoxicidad causada por doxorrubicina y no los daños causados por 5-fluoracilo.

Palabras clave: Cardiotoxicidad, yodo, doxorrubicina, 5-fluoracilo.

\section{Introducción}

El yodo es un micro nutrimento esencial para la integridad de la glándula tiroides y la síntesis de hormonas tiroideas. En humanos la concentración total de yodo es de 30 a $50 \mathrm{mg}$, en menos de $30 \%$ se encuentra en esta glándula e incorporando a sus hormonas, del 60 al $80 \%$ se encuentra en tejidos extratiroideos y su papel biológico aun se desconoce. Los requerimientos de yodo en el humano

* Departamento de Medicina. Universidad Autónoma de Aguascalientes.

** Instituto de Neurobiología de la UNAM, Campus Juriquilla. 
oscilan entre 100 y $200 \mu \mathrm{g}$, los cuales son para satisfacer la función tiroidea; pero se necesitan de 3 a $5 \mathrm{mg}$ /día para cumplir con su papel antioxidante 1 .

Los radicales libres son elementos químicos con un único electrón desapareado en la órbita externa por lo que son muy inestables y altamente reactivos ${ }^{2}$. Los radicales libres $(R L)$ cumplen una importante función en procesos homeostáticos como intermediarios en reacciones de oxidación-reducción (redox) pero las cantidades excesivas son tóxicas ya que alteran la estructura y función de macromoléculas como lípidos, proteínas y ADN, involucrándose en neoplasias, procesos inflamatorios y otras patologías. Experimentos recientes han demostrado que el yodo es un antioxidante muy potente, sin embrago se desconoce el mecanismo exacto mediante el cual el yodo ejerce su efecto antioxidante, se sugieren dos posibles mecanismos: 1) el yodo actúa como un donador de electrones estabilizando a los RL. 2) compitiendo con los RL por los sitios altamente oxidables de las moléculas antes mencionadas, evitando las reacciones oxidativas en cadena ${ }^{1}$.

La doxorrubicina (dox) es un agente citotóxico del tipo de las antraciclinas utilizado como antineoplásico en una gran variedad de tumores malignos; sin embargo este efecto ha sido opacado por su efecto cardiotóxico, debido principalmente a la producción de radicales libres y al stress oxidativo que provoca ${ }^{3}$. El corazón es vulnerable al daño que causa ya que la actividad antioxidante de este órgano es muy escasa, debido probablemente a su baja actividad de catalasa ${ }^{4}$. Estudios en ratas demuestran que la administración de naringenina, un flavonoides antioxidante, disminuye la cardiotoxicidad inducida por este fármaco ${ }^{3}$. Por lo que se propone la incorporación a la quimioterapia de agentes antioxidantes como el yodo para disminuir la cardiotoxicidad inducida por dox.

La incidencia de cardiotoxicidad por 5-fluoracilo (5-FU) oscila entre el 1.2 a $18 \%$ de los pacientes que reciben este medicamento, clínicamente el daño se manifiesta como síndromes coronarios agudos, caracterizados por dolor precordial, arritmias, alteraciones electrocardiográficos compatibles a cambios isquémicos, elevación de enzimas cardíacas o incluso falla cardíaca aguda ${ }^{5,6}$. Los estudios histopatológicos han demostrado hemorragias intersticiales miocárdicas, necrosis multifocal de miofibrillas, reacciones inflamatorias, cambios vasculares, y de una vacuolización similar a la encontrada en la cardiotoxicidad por dox. Sin embargo poco se conoce del mecanismo de daño inducido por 5-FU, algunos autores proponen al espasmo de arterias coronarias como la causa de la isquemia y toxicidad cardíaca producida por 5-FU7.

El objetivo de este trabajo consiste en valorar y comprobar el efecto antioxidante del yodo como protector en la cardiotoxicidad inducida por doxorrubicina y determinar el mecanismo de lesión cardiaca inducida por 5-fluoracilo. 


\section{Material y métodos}

\section{Material biológico:}

Se utilizaron 18 ratas hembras vírgenes de la cepa Sprague-Dawley de 6 semanas de edad. Los animales crecieron en el bioterio del Instituto de Investigaciones Biomédicas bajo condiciones de temperatura $\left(18-22^{\circ} \mathrm{C}\right)$ y luz-oscuridad (12:12) controladas con una dieta adlibitum a base de Purina Rat Chow; con un peso promedio de $143 \mathrm{~g}$. Se dividieron en los siguientes seis grupos: 1) control, 2) Yodo, 3)Dox, 4)Dox mas Yodo, 5)5-FU y 6)5-FU mas Yodo, cada grupo con una $\mathrm{n}=3$.

La doxorrubicina en ámpula (Adriblastina RD, Pharmacia \& Upjohn) se administró dos veces (tercer y séptimo día) a dosis de $1 \mathrm{mg}$ intraperitoneal. El 5-FU (Fluorox) se administró a dosis de $1 \mathrm{mg}$ intraperitoneal durante cuatro días consecutivos (cuarto al séptimo día). Para la suplementación con yodo molecular se usó lugol al $0.05 \%$ en agua adlibitum (del tercer al octavo día). El grupo control no recibió tratamiento.

Los animales se sacrificaron mediante el método de decapitación, se disecó el corazón se guardó y homogenizó en un buffer que contiene sacarosa $225 \mathrm{mM}$, Tris/ $\mathrm{Hcl} 10$ $\mathrm{nM}, \mathrm{BSA} 0.2 \%$, EGTA $0.3 \mathrm{mM}$ : pH 7.4 .

\section{Determinación de catalasa en sue- ro y corazón}

La actividad de la catalasa se midió mediante el método de Hugo Aebi. La actividad fue expresada en umol/min/ umg de proteína. La cuantificación de proteína se llevó a cabo mediante el método de Bradford.

\section{Lipoperoxidación en corazón y suero}

La concentración de estos compuestos fue cuantificado mediante la reacción de ácido tiobarbitúrico (TBA) y expresada como nanomoles de malondialdehido (MDA)/mg de proteína. La técnica nos permite detectar el índice de oxidación basal.

\section{Cuantificación de p53}

Se aisló el ARN total mediante método de Trizol y se cuantificó con un espectrofotómetro a una longitud de onda de $260 \mathrm{~nm}$. Mediante la técnica de reversotranscripción se llevó a PCR en tiempo real.

Método estadístico.

El análisis estadístico de los resultados se realizó con ANOVA de una vía y la prueba poshoc de Tukey.

\section{| | | | | | | | | | | | | | | | | | | | | | | | | | | | | | | | | | | | | | | | | | | | | | | | | | | | | | | | | | | | | | | | | | | | | | | | | | | | | | | | | | | | | | | | | | | | | | | | |}

\section{Resultados}

En la figura 1 se muestra la actividad de catalasa en corazón en la que se aprecia que los grupos que recibieron tratamiento con dox y 5-FU tienen tendencia a disminuir la actividad de la enzima, mientras que el grupo de dox mas yodo aumenta significativamente la actividad de esta enzima con respecto al grupo dox, sin embargo no se observa dicho fenómeno con el grupo 5-FU mas yodo con respecto al grupo 5FU y se aprecia que el grupo de ratas que recibieron únicamente yodo no se aprecia modificación alguna con respecto al grupo control. En la figura 2 se muestra la lipoperoxidación en corazón en la que se observa que no hay diferencia significativa de todos los grupos con respecto al grupo control. Dox con respecto al grupo control muestra una tendencia a aumentar los niveles de lipoperoxidación, y las ratas que recibieron adicionalmente yodo tienden a disminuirlos niveles de lipoperoxidación encontrados en el grupo dox. No se encontraron cambios significativos entre los grupos 5-FU y 5-FU más yodo. En la figura 3 se muestra la cuantificación de p53 en la que se observa que todos los grupos que recibieron yodo tienden a disminuir el número de copias de este gen, al contrario de los grupos que recibieron la quimioterapia en donde tienden a aumentarse con respecto al grupo control. 

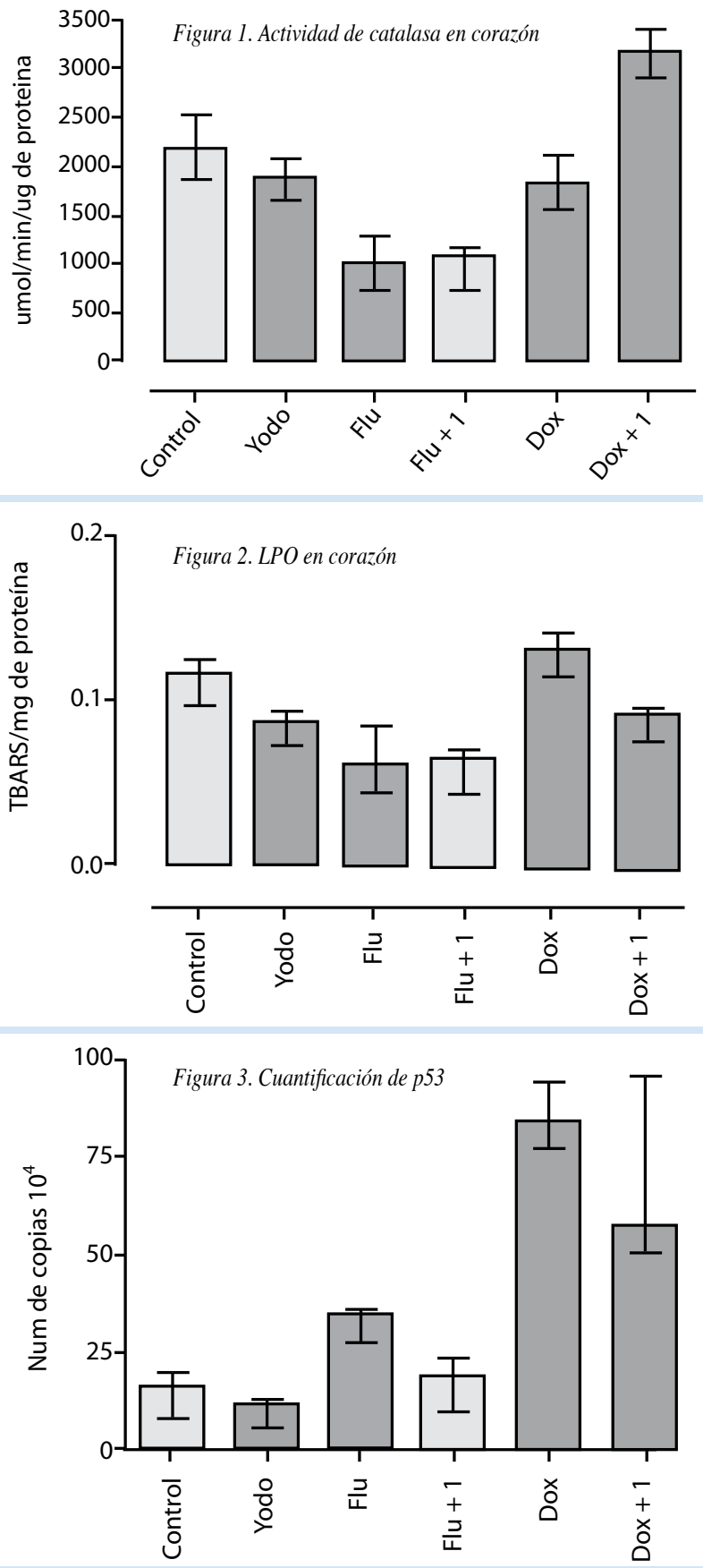

Discusión

En el presente trabajo mostramos que la administración de yodo puede reducir la cardiotoxicidad inducida por doxorrubicina, ya que la adición de yodo a la quimioterapia aumenta la actividad de catalasa y disminuye los índices de lipoperoxidación. El yodo no tiene el mismo efecto protector en la quimioterapia de fluoracilo, esto se podría explicar debido a que probablemente la toxicidad que ejerce fluoracilo no es mediante la producción de radicales libres, sino que produce vasoespasmo de la arterias coronarias como lo menciona Tsibiribi y colaboradores ${ }^{8}$.

También nuestros resultados muestran que el yodo tiende a disminuir la expresión de p53, esto indica que probablemente es capaz de reducir el daño celular causado por quimioterapia, aunque el mecanismo mediante el cual lo hace aún es desconocido ${ }^{1}$.

Los mecanismos mediante los cuales el yodo ejerce su poder antioxidante aún se desconocen, sin embargo en base a los resultados aquí obtenidos se proponen las siguientes posibilidades: 1) el yodo actúa como un donador de electrones estabilizando los radicales libres y 2) el yodo compite con los radicales libres por los sitios altamente oxidables de las moléculas, como lípidos, proteínas o ADN, evitando las reacciones oxidativas en cadena.

En conclusión nosotros encontramos que: La doxorrubicina tiende a aumentar los niveles de lipoperoxidación y a disminuir los de catalasa en el corazón. Y que el yodo es un antioxidante cuyo efecto reduce la toxicidad cardíaca inducida por doxorrubicina.

La administración de yodo no influye en el tratamiento con 5-fluoracilo.

El yodo en un futuro podría utilizarse como protector en diferentes patologías que son producto de la formación de una cantidad excesiva de radicales libres.

\section{Bibliografía}

1 Aceves C, Anguiano B, Delgado G. Is iodine a gatekeeper of the integrity of the mammary gland?. Journal of Mammary Gland Biology and Neoplasia 2005; 10:189-196.

2 Robbins MD. Patología Estructural y Funcional. Editorial Mc Graw Hill. España 2000.

3 Arafa HM, Abd-Haled MF, Hafez MF. Abatement by naringenin of doxorubicin-induced cardiac toxicity in rats. Journal of the Egyptian Nat. Cancer Inst. 2005; 17:291-300.

4 Kang JY. Chen Y, Epstein PN. Supression of doxorubicin cardiotoxicity by over expression of catalase in the heart of transgenic mice. J. Biol. Chem. 1996;271:12610-12616.

5 Kumar S, Gupta RK, Somal N. 5-Flouracil induced cardiotoxicity in albino rats. Mater Med Pol. 1995;2:6366.

6 Tsibiri P. Cardiac lesions induced by 5 -fluoracil in the rabbit. Hum Exp Toxicol. 2006;6:305-309.

7 Dazell JR, Samuel LM. The spectrum of 5-fluorouracil cardiotoxicity. Anticancer Drugs 2009;20(1):79-80.

8 Tsibiri P. Cardiotoxicity of 5-fluoracil in 1350 patients with no prior history of heart disease. Bull Cancer. 2006;3:E27-E30 